\title{
42 years Old Female with History of Epigastric Pain and Jaundice
}

\author{
Sushila B Ladumor ${ }^{1 *}$, Adham Darweesh ${ }^{2}$ and Hiba Esmayil ${ }^{3}$ \\ ${ }^{1}$ Consultant Radiologist, Assistant Professor in Clinical Radiology, Weil Cornel Medical College, Qatar \\ ${ }^{2}$ Senior Consultant Radiologist, Hamad Medical Corporation, Qatar \\ ${ }^{3}$ R1 Radiology Resident, Hamad Medical Corporation, Qatar
}

Received: September 04, 2017; Published: September 18, 2017

*Corresponding author: Sushila B Ladumor, Consultant Radiologist, Hamad Medical Corporation, HGH, Clinical Imaging, P.O. Box 3050, Doha, Qatar Assistant Professor in Clinical Radiology, Weil Cornel Medical College, Doha, Qatar, Email: drsbladumor@yahoo.com

\begin{abstract}
Choledocholithiasis denotes the presence of gallstones within the bile ducts (common hepatic duct/ common bile duct). US is best choice of investigation for the patient with history of epigastric pain and jaundice as initial work up along with clinical and laboratory exam as it is easily available, cheap, non-invasive and no radiation. Magnetic resonance (MR), cholangiopancreatography (MRCP) is increasingly being used to evaluate pancreatobiliary disease, providing a noninvasive alternative to endoscopic retrograde cholangiopancreatography (ERCP) [1]. Many reports have described its usefulness and diagnostic accuracy in evaluating various pancreatobiliary abnormalities [2-3], and, according to some reports, in many instances MRCP can replace diagnostic ERCP.
\end{abstract}

Keywords: US; MRI; MRCP; Post Contrast; Stone; Dilatation; Accosting shadowing; MPD

Abbreviation: US: Ultrasound; CBD: Common Bile Duct; IHBR: Intra Hepatic Biliary Radicals; MPD: Main Pancreatic Duct; MRCP: Magnetic Resonance Cholangiopancreatography, ERCP: Endoscopic Retrograde Cholangiopancreatography; EUS: Ultrasonography; MR: Magnetic Resonance

\section{Clinical History}

42 years Old Female with History of Epigastric Pain and Jaundice, Ultrasound abdomen requested to rule out cholecystitis.

\section{Discussion}

\section{Clinical presentation}

Stones within the bile duct are often asymptomatic and may be found incidentally however, more frequently they lead to symptomatic presentation with:
a. Biliary colic
b. Ascending cholangitis
c. Obstructive jaundice
d. Acute pancreatitis

\section{Pathology}

Stones within the bile duct may form either in situ or pass from the gallbladder, and when recurrent tend to be pigment stones, and are thought to be associated with bacterial infection.

\section{Radiographic features \\ Ultrasound}

Although ultrasound is usually the first investigation for biliary disease, it has average sensitivity for the detection of biliary stones within the bile duct. Sensitivity has been variably reported between $13-55 \%$, with newer studies having higher values due to improved equipment. CBD must be evaluated completely up to distal end when it passes through the pancreatic head by longitudinal and transverse scan fully looking stone or abnormal mass (Figure 1).

\section{Findings}

US: Detection of biliary stones depends in part on technologic factors and in part on the care taken by the interpreting physician. Include visualization of stone or echogenic rounded focus (sludge) with size ranges between $2 \mathrm{~mm}$ reaching up t more than 20 $\mathrm{mm}$. Posterior shadowing may be more difficult to see than with gallstones within the gallbladder as more than $20 \%$ of common bile duct stones will not shadow. Dilated bile duct more than $6 \mathrm{~mm}+1$ 
$\mathrm{mm}$ per decade above 60 years of age and more than $10 \mathrm{~mm}$ post cholecystectomy with dilated intrahepatic biliary tree. Presence of multiple small gallstones should raise suspicion of CBD stone when no clear CBD stone seen by US Recently endoscopic ultrasonography (EUS) has also been used with very high sensitivity and specificity (Figure 2).

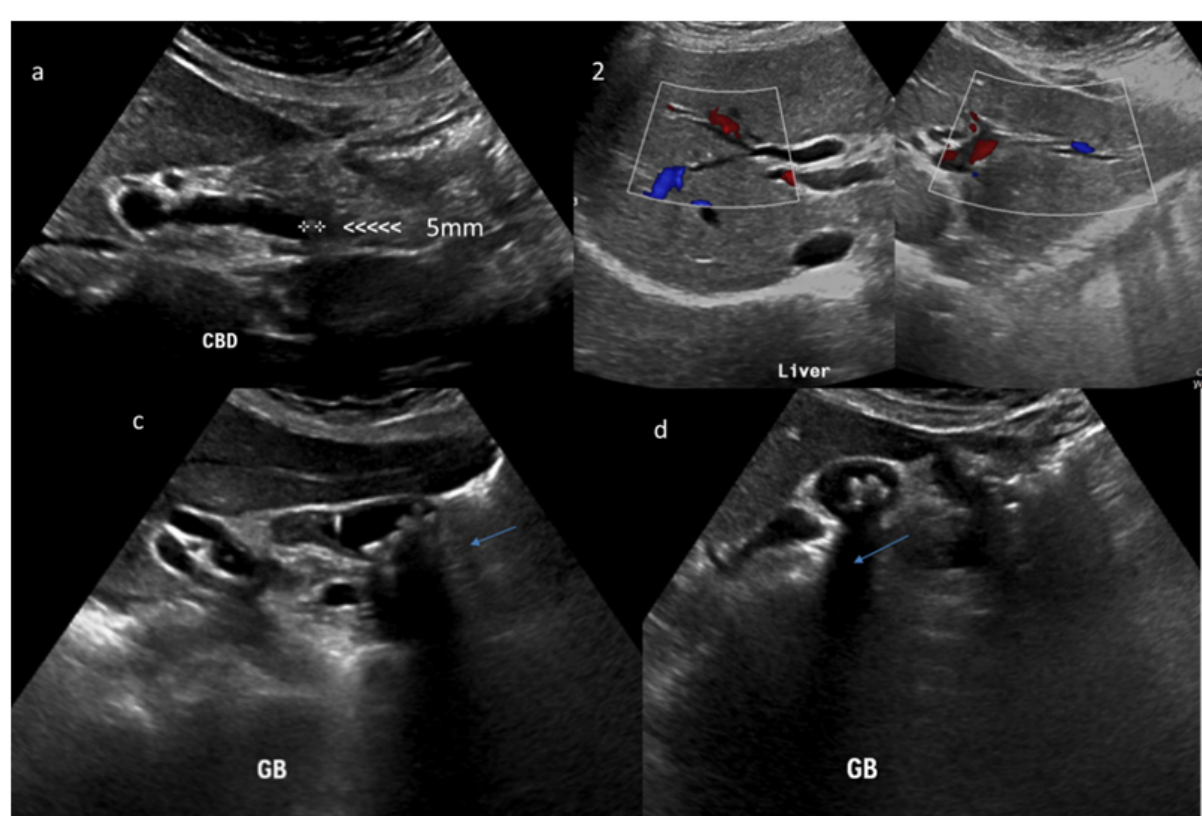

Figure 1: US: Dilated CBD with stone in distal CBD measures about $5 \mathrm{~mm}$ (shown by arrow head with measurement in image (a), Mildly dilated central intra hepatic biliary radicles (b), Multiple stones in gall bladder showing strong posterior acoustic shadowing (c, d). Nopericholecystic fluid. Gall bladder wall is not thickened.

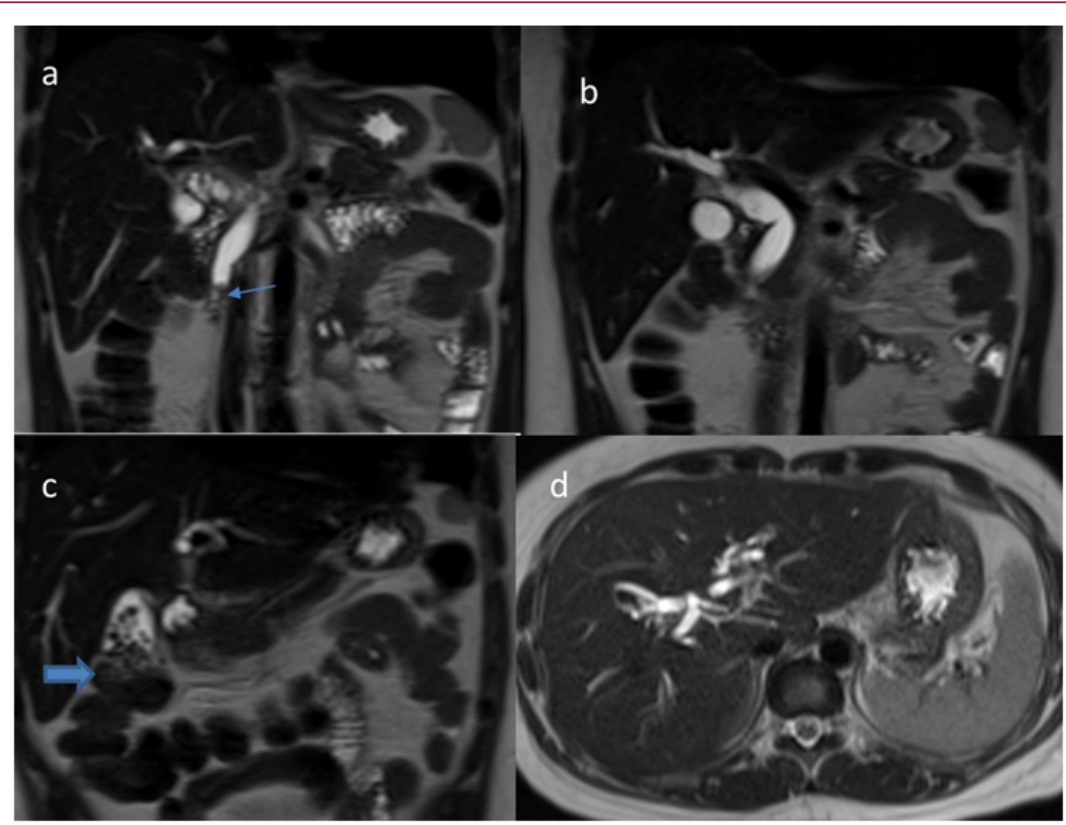

Figure 2: T2 weighted coronal $(\mathrm{a}, \mathrm{b}, \mathrm{c})$ and axial $(\mathrm{d})$ demonstrates dilated CBD and mildly dilated intrahepatic biliary radicles. Filling defect in distal CBD shows T2 low signal (a) representing stone. Multiple small T2 low signal filling defect in GB (c) representing multiple GB stones same as US.

CT: Routine contrast enhanced CT is moderately sensitive to choledocholithiasis with a sensitivity of $65-88 \%$ [3], but requires attention to a number of potentially subtle findings including target sign, central rounded density (stone), surrounding lower attenuating bile or mucosa or calcification of the stone: unfortunately only $20 \%$ of stones are of high density and better seen in unenhanced CT along with dilated biliary tree in absence of appreciable focal mass. Setting window level to the mean of the bile duct and setting the window width to $150 \mathrm{HU}$ has been reported to improve sensitivity (Figure 3). 


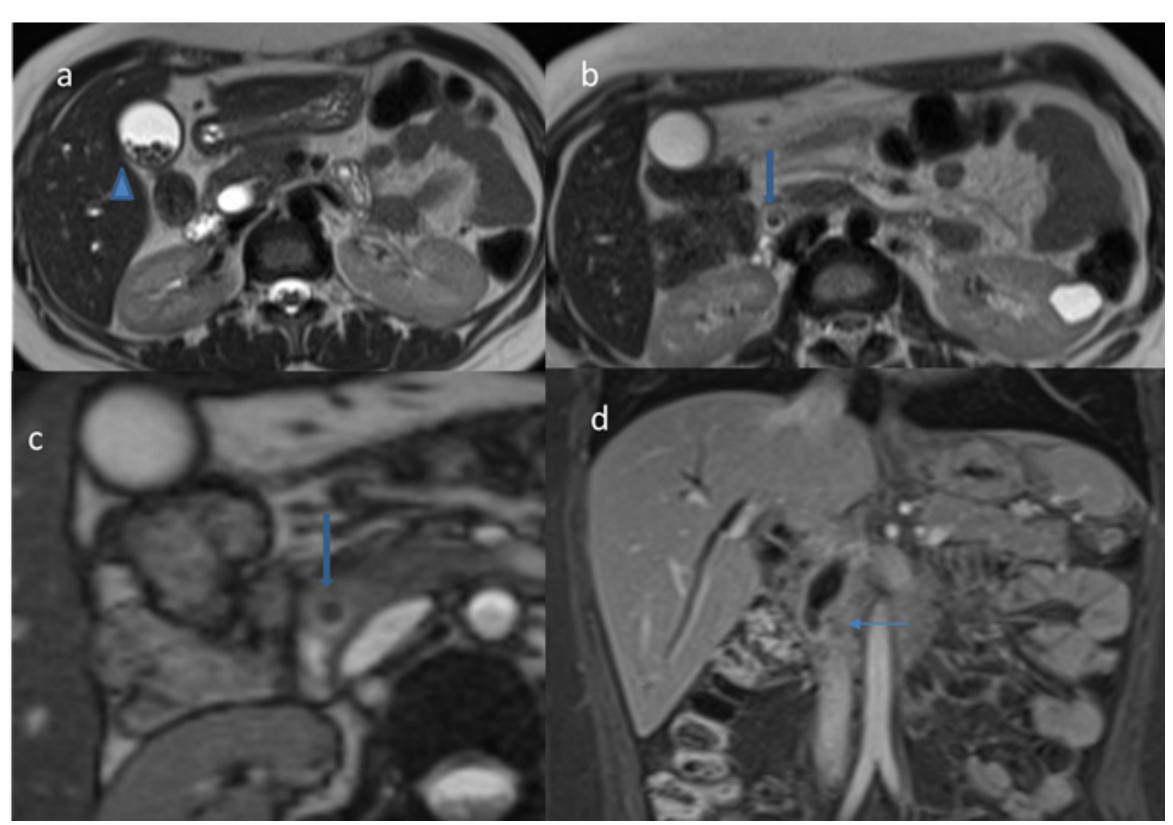

Figure 3: T2 weighted axial Image $(\mathrm{a}, \mathrm{b}, \mathrm{c})$. Multiple GB stones (a), Low signal filling defect in distal CBD (b, c) representing distal CBD stone. Post contrast coronal image in late Porto venous phase shows dilated CBD and IHBR with mildly thick enhancing wall of distal CBD likely due to secondary inflammation due to obstruction. No focal liver lesion. Incidentally noted left renal cyst (b).

MRCP: Magnetic Resonance Cholangio pancreatography (MRCP) has largely replaced ERCP as the gold standard for diagnosis of choledocholithiasis, able to achieve similar sensitivity (90-94\%) and specificity (95-99\%) without ionizing radiation, intravenous contrast, or the complication rate inherent in ERCP.Filling defects are seen within the biliary tree on thin cross-sectional $\mathrm{T} 2$ weighted imaging. Care should be taken not to use thick slabs for the diagnosis as volume averaging may obscure smaller stones [1]. When dilated bile ducts are found, a search for the cause of obstruction should be done seriously and should include correlation with clinical symptoms and serum markers (Figure 4). Possible causes of bile duct obstruction include biliary strictures, stones, inflammation, and neoplasm. Although the differentiation of a benign from a malignant stricture is sometimes difficult [1-3].

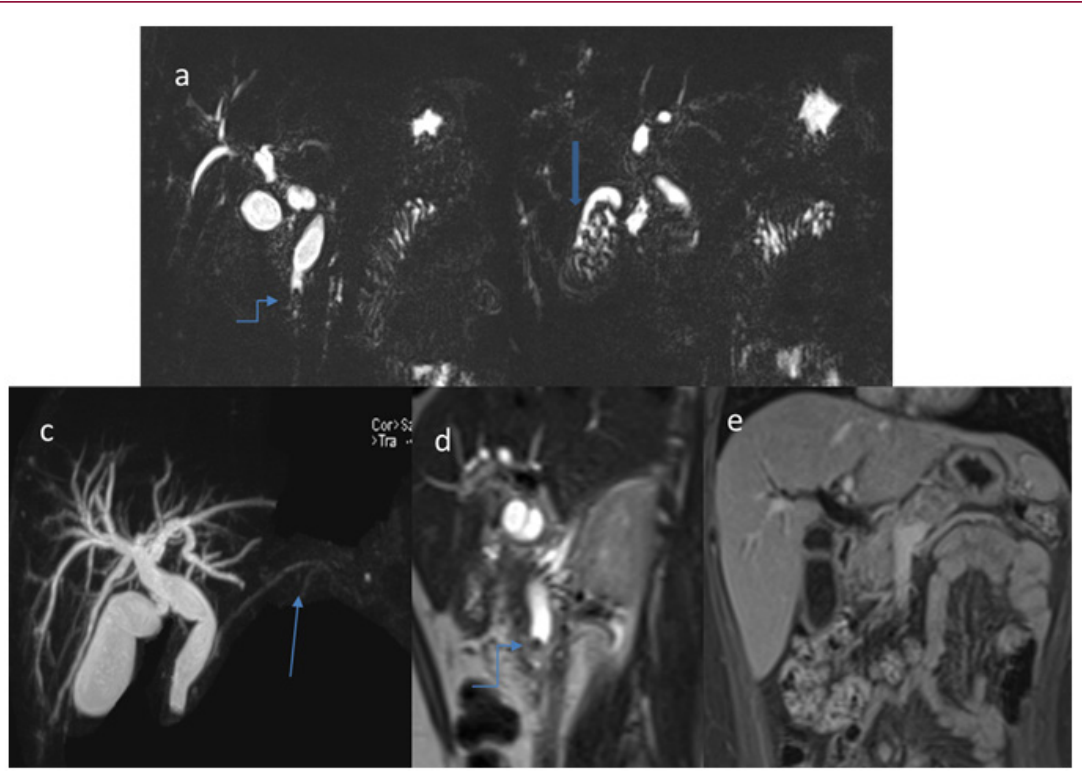

Figure 4: $\operatorname{MRCP}(a, b, c)$ demonstrates dilated CBD and IHBR with filling defect (stone) in distal CBD (Curved arrow in a, d) Pancreatic duct is not dilated (thin arrow in image c). Post contrast late Porto venous coronal image shows normal GB wall. No pericholecystic fluid. No definite CBD mass. No area of restricted diffusion. No abnormal enhancing focal distal CBD mass. Pancreas, liver, spleen, adrenals and kidneys (apart from simple Bosniak type I left renal cyst as well as vascular structures are unremarkable (All images with organs are not shown here). 


\section{Treatment and Prognosis}

Endoscopic Retrograde Cholangiopancreatography (ERCP) with sphincterotomy is the treatment of choice for choledocholithiasis. Acute pancreatitis is commonest complications of ERCP and sphincterotomy.

\section{Differential Diagnosis}

There is usually little differential, and differential will depend on the modality. The most frequent entities to consider include:

a. Malignancies

b. Cholangiocarcinoma

c. Ampulla of Vater carcinoma: arises from a distal position, at the pancreaticobiliary junction, and has low attenuation.

\section{d. Pancreatic Aden carcinoma}

Other filling defects could be airbubbles, parasites mimics as partial volume averaging of bowel gas, vascular calcification, and surgical clips where MRCP is most help full for confirmation of stone and MRI with contrast along with restricted diffusion to exclude malignancy [2,3].

\section{Diagnosing Stone with Other Differential and Mimics of Stone}

First, biliary stones tend to be in a dependent posterior location in the biliary tract, and a crescent of bile or gas is commonly seen outlining the anterior portion of the stone. Second, stones are often geometric and angulated in shape. Third, stones frequently have a lamellated appearance, particularly at CT and Fourth signs of inflammation such as periductal edema, biliary epithelial thickening, and mural enhancement may point to local irritation caused by stones, or to associated cholangitis or cholecystitis. However, mural enhancement has been reported to be frequently seen with malignancy and should prompt careful investigation [3].

\section{Conclusion}

Improved US, MR imaging and CT diagnosis of biliary disease can be achieved with detailed knowledgeable, detailed search of the duct lumen, duct wall, and surrounding structures, as well as an understanding of imaging technique is extensively helpful in reaching proper diagnosis.

\section{References}

1. Yeh BM, Liu PS, Soto JA, Corvera CA, Hussain HK (2009) MDMR Imaging and CT of the Biliary tract. Radiographics 29(6): 1669-1688.

2. Choi SH, Han JK, Lee JM, Lee KH, Kim SH, et al. (2005) Differentiating malignant from benign common bile duct stricture with multiphasic helical CT. Radiology 236(1): 178-183.

3. Park MS, Kim TK, Kim KW, Park SW, Lee JK, et al. (2004) Differentiation of extrahepatic bile duct cholangiocarcinoma from benign stricture: findings at MRCP versus ERCP. Radiology 233(1): 234-240.

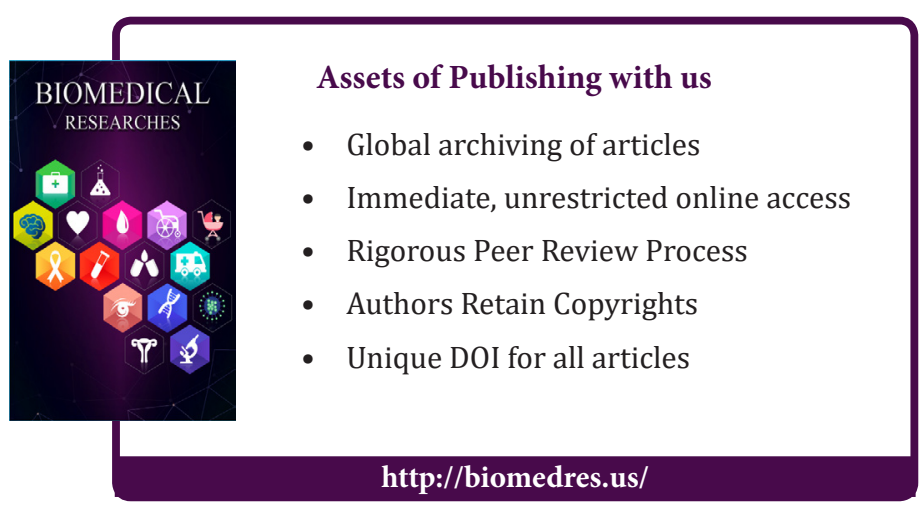

